\title{
GASTROINTESTINAL MICROBIOTA AND IMMUNITY.
}

\author{
Kroum K. Hristov \\ Department of Chemistry and Biochemistry, Faculty of Medicine, Medical \\ University - Sofia, Bulgaria..
}

\begin{abstract}
:
There is a critical time-window early in life during which appropriate microbial exposure has to occur in order to induce functional, life-long immune regulation. Actinobacteria and Proteobacteria dominate the GIT microbiota of children. With age, Firmicutes and Bacteroidetes dominate the commensal's landscape. Proteobacteria are most abundant in GIT of elderly people. A critical level of microbial diversity after birth is required to prevent the induction of hyper-IgE during adulthood. The colonisation process induces a change from foetal $\mathrm{TH} 2$ to a TH1/TH17 T-cell state. There is a temporal activation of the innate mucosal immunity: TLR2 and TLR4 expressed in the sterile foetal environment are replaced by TLR5, coinciding with the post-natal microbial expansion; as TLR tolerance develops, it attenuates and remain diminished into adulthood. CD5+ B-cells contribute to the first line of defence against bacterial infections. Ageing is associated with an increased inflammatory response via the CD14/TLR4/MD2 receptor complex (monocytes, DC, macrophages, and B-cells), and increased ROS production (neutrophils). Gut flora changes post-mortem, presenting as biphasic pattern of bacterial overgrowth (after 3 and $72 \mathrm{hr}$ post-mortem). Intestinal bacteria translocateto MLN, spleen, liver, kidney, and cardiac blood, as early as 5 min after death. Intestinal tissue destruction is observed within $3 \mathrm{hr}$ post-mortem, whereas proliferative lymphocytes decrease between 3 and $12 \mathrm{hr}$ post-mortem. The stomach microbiota is less diverse than that in mouth, colon, and stool. H. pylori induces chronic inflammation of the stomach mucosa. Colonisation resistance comprises the ability of GIT microbiota to deter pathogens from causing infections. Probiotics (Bifidobacteria and Lactobacilli), prebiotics (DHNA), and dietary supplements (arginine) modulate intestinal commensals, maintaining the homeostasis, and conferring a health benefit on the host.
\end{abstract}

Keywords: gastro-intestinal microbiota, mucosal immunity, ageing, innate immunity, B-cells, T-cells,

\section{BACKGROUND:}

The bacteria colonising the human gastro-intestinal tract comprise a functional unit, consisting of 10-times more cells and 100-times more genes than the human genome, and has the metabolic capacity of the liver. GIT microbiota is a structure with bi-directional regulation in health and disease. The microbiota influences the post- natal development of the immune system, such that deviations due to Caesarian section, formula-based diet, hygiene, vaccination, antibiotics, and antimicrobials, after the birth to adulthood. Microbial community dynamics demonstrates high temporal consistency within and distinct composition between individuals. It is shaped by the host immunoregulatory phenotype.

\section{Birth, neonates, and elderly:}

The sterile newborn intestine is colonised by environmental microorganisms after birth that expand exponentially to numbers that exceed the total numbers of host mammalian cells (Fig. 1. Microbiota activates NF-kappaB in GIT). GIT microbiota is shaped by the host immunity, the environment, and the diet. Microbiota contributes to IBD, obesity, malnutrition, autoimmunity, and allergic asthma. Immunodeficiencies (Wiskott-Aldrich syndrome, Omenn syndrome, immunodysregulation poly-endocrinopathy enteropathy X-linked syndrome) result in dysbiosis that causes increased IgE levels (playing a central role in atopic allergic disease and immunity to parasites). Similarly, murine immunodeficiency models, and CD4 KO, MHCII KO, and athymic nude mice display high IgE.

The colonisation process induces a switch from foetal TH2 T-cell state (evolved to avoid intra-uterine maternal rejection) to a $\mathrm{TH} 1 / \mathrm{TH} 17 \mathrm{~T}$-cell state (preventing invading pathogens). While commensals have little effect on systemic T-cell numbers, they increase post-natal Bcell numbers in both MLN and spleen (B1 B-cell progenitors in secondary follicles within GC, and B2 B-cells in PP and MALT, not present at birth). There is a temporal activation of the innate mucosal immune processes that rapidly attenuates as the host ages. While TLR2 and TLR4 (sensing peptidoglycans and LPS, respectively) are expressed in the sterile foetal environment, TLR5 (sensing flagellin) is highest in 2-week-old mice, coinciding with the post-natal microbial expansion in the GIT. It attenuates in the 3-weeks-old (as TLR tolerance develop), and remain diminished into adulthood [1].

B-cell differentiation (memory B-cells) and activation (IgM to IgA IS, and lumenal Ab secretion) depend on intestinal commensals colonisation after birth (E. coli, and/ or Bifidobacteria). In humans, CD5+ B-cells (producing lowaffinity polyreactive IgM that cross-react with bacterial Ag, including LPS) arise early in ontogeny and are the major B- 
cell population in cord blood, and during the first years of life, after which they decrease in numbers. CD5+ B-cells contribute to the first line of defence against bacterial infections. At birth, boys have a significantly higher fraction of B1 B-cells, compared with girls [2].

Microbiota is essential for the control of basal $\operatorname{IgE}$ levels. IgE levels increase after day 30 of age, simultaneously with the transition from lactation to solid food. A critical level of microbial diversity within 1 week post-weaning is required to inhibit the induction of hyper- $\operatorname{IgE}$ during adulthood. The neonatal IgE IS takes place in the mucosal sites - PP and MLN (not in the BM, spleen, PLN, and peritoneal cavity B1 B-cells), and is regulated by CD4+ T-cells, IL4, IL7, IL10 and IL13 [3]. Broad-spectrum antibiotics reduce the biodiversity of fecal microbiota and may be a factor causing NEC. In the absence of commensal microbiota, mice have decreased $\operatorname{IgA}$, reduced intraepithelial lymphocytes, reduced PP and MLN size and cellularity, decreased responses to T-cell mitogens, and altered susceptibility to pathogenic organisms. MR mice have increased bacterial translocation across the intestinal epithelial barrier (GFPlabeled E. coli are detected in MLN, spleen, and liver), and fewer splenic B-cells, and MLN CD4+ T-cells, but normal numbers of splenic T-cells. MR mice maintain the foetal TH2 phenotype (regulated by progesterone to prevent foetal rejection, and expressing more IL4 and IL10), and fail to develop TH1/TH17 T-cells, essential for the anti-microbial defence (less IFN-gamma and IL17). MR mice have decreased TLR expression, and increased intestinal injury similarly to TLR2 KO and/or TLR4 KO mice. MR mice express significantly less neutrophil-attracting chemokines, MIP2, CXCL2, LIX, and CXCL5 [1].

Establishing Lactobacilli population early in life maintain and repair mucosal barrier functions, inhibiting the growth of $\mathrm{pH}$-sensitive E. coli O157:H7, Staphilococcus aureus, Bacillus cereus, and Clostridium perfringes, and decreases the expression of CCL2, CCL8 (IL8), CCL28, CCR1, and CXCR4 during decreased microbiota diversity [4]. Oligosaccharides in human milk account for the difference of GIT microbiota (Lactobacilli and Bifidobacteria) between breast-fed and formula-fed infants.

Polydextrose is a candidate prebiotic, developed as a bulking agent for foods, increases ileal Lactobacilli, and improves allergy and infection symptoms (decreasing ileal TNF-alpha, IL1-beta, IL8, and IL10 expression, and increasing FcRn expression, extending IgG half-life and its intestinal delivery) [4].

Bifidobactera and Enterobacteria dominate the GIT microbiota of children. With age, and during adulthood, Firmicutes (Clostridiaceae, Blautia, Faecalibacterium, Lactobacillus) and Bacteroidetes (Bacteroides) dominate the commensal's landscape. The adult bacterial composition shows temporal (morning/afternoon), and diet fluctuations [5]. New changes appear in senescence when microbiota of elderly people is dominated by Proteobacteria [6]. The changes in the bacterial populations in elderly represent the loss of health-associated and youth-associated microbiota components, and gain of an elderly-associated microbiota. The microbiota of elderly ( $>65$ years old) show greater inter-individual variation. Centenarians have different and less diverse microbiota, with an increased facultative anaerobes (Proteobacteria and Bacilli), and a decrease of Bacteroides, Bifidobacterium and Enterobacteriaceae, and presence of specific bacteria (Faecalibacteriumprauznitzii and Clostridium cluster XIVa) [5]. Distinctive GIT microbiota configurations are identified in both community (healthy high-fibre diets: Coprococcus, Prevotella, and Catenibacterium), and long-stay residential care elderly subjects (Anaerotruncus, Desulfovibrio, and Coprobacillus). Age and long-term care are associated with a diet low in plant-based nutrients and high in saturated fat and sugar, as well as coresidence with other elderly individuals, high antibiotic treatment rates, and increased co-morbidity. The high consumption of plant-based high-fibre foods correlates with diverse microbiota, associated with healthy ageing, although higher microbiota diversity is not linked with superior health. The diet has a cumulative effect over time, causing an initial rapid change in state [7].

Ageing is closely associated with chronic inflammatory states (redox imbalance), leading to chronic inflammatory and degenerative diseases (IBD, rheumatoid arthritis, Alzheimer's disease, and cardiovascular disease). Ageing results in increase of Firmicutes and Tenericutes, as well as reduction of Bacteroidetes and Prevotellaceae. Facultative anaerobes (Streptococci, Staphylococci, Enterococci, and Enterobacteria) increase. Ageing is associated with increased production of LPS, stimulating the inflammatory response via the CD14/TLR4/MD2 receptor complex(monocytes, DC, macrophages, and B-cells), and increased ROS production via NADP oxidase activation (neutrophils). While M1 (classically-activated, secreting pro-inflammatory TNF-alpha and IL6) are higher in the colons of aged rats, M2 (alternativelyactivated, secreting anti-inflammatory cytokines, MRC1) are lower. Lactic acid bacteria (Lactobacillus pentosus sp. plantarum C29, isolated from fermented vegetables) have a beneficial effect on age-dependent inflammation, inhibiting TNF-alpha, IL6, ROS production, and the activation of NF-kappa-B, MAPK, and AP1. Treatment with C29 reduces the expression of tight junction proteins ZO1, occludin, and claudin-1, and reduces the intestinal microbial and plasmatic LPS levels, as well as the Firmicutes to Bacteroidetes ratio, which is significantly higher in aged rats. C29 suppresses age-dependent colitis, restoring age-disrupted gut microbiota composition and LPS production to those of young rats [8]. Gut flora changes post-mortem. The ileal overgrowth starts 3 and $24 \mathrm{hr}$ post-mortem. The obligate anaerobes (Bacteroides/Prevotella spp., Clostridium coccoides, and C. leptum, as well as mouse intestinal Bacteroides) follow a biphasic detection pattern: increase within $3 \mathrm{hr}$, and decline to baseline at $12 \mathrm{hr}$, followed by a second increase after $72 \mathrm{hr}$ post-mortem. While Bifidobacteria increase until $6 \mathrm{hr}$ and drop back to baseline at $24 \mathrm{hr}$, the total intra-luminal colonic bacterial loads decrease between 3 and $6 \mathrm{hr}$ post-mortem. Total ileal eubacterial load (Enterobacteriaceae, Enterococci, and Lactobacilli) is significantly higher after $72 \mathrm{hr}$ post-mortem. Intestinal bacteria translocate to MLN, spleen, liver, kidney, and cardiac blood, as early as $5 \mathrm{~min}$ after death. Intestinal tissue destruction (apoptotic 
cells and neutrophils) is observed within $3 \mathrm{hr}$ post-mortem, whereas proliferative lymphocytes (T- and B-cells) decrease between 3 and $12 \mathrm{hr}$ post-mortem. Interestingly, at $1 \mathrm{hr}$ postmortem, bacterial translocation rates are lowest in all compartments, with vitally no bacterial growth in the spleen, liver, kidney, and blood. The nadir of the total load at 30 min post-mortem could be observed when quantifying Enterobacteria, Enterococci, Clostridia and Bifidobacteria, but not Lactobacilli and Bacteroides/ Prevotella spp. The nonlinear "undulating" pattern of bacterial numbers in HFA mice post-mortem is a consequence of the decreasing body core temperature, accumulation of toxic products, and shortage of nutrients and oxygen. Thereafter, the bacteria adjust their metabolism and get accommodated to the new environmental conditions [9].

Microbiota - mouth, oesophagus, stomach, duodenum, ileum, colon:

Although beneficial in the GIT of humans, Lactobacillus spp. are associated with tooth decay and cavities in the mouth.

Unlike humans, mice have relatively high gastric $\mathrm{pH}$ 3-4, which decreases the potential to inactivate microorganisms. Murine gastric microbiota is dominated by Lactobacillus spp. In contrast, mongolian gerbils and guinea pigs, similarly to humans, have low intragastric $\mathrm{pH}<2$. Lactobacillus, Streptococcus, and Propionibacterium can regularly be sampled from the stomach of healthy adults. The stomach microbiota (Lactobacillus sp., Veillonella sp., Clostridium sp., and Corynebacterium sp.) is less diverse than the bacterial communities in mouth, colon, and stool. There is a positive correlation between Spirochaetae and H. pylori, and negative correlation between $H$. pylori and Bacteroidetes, Chloroflexi, Cyanobacteria, Fusobacteria, Plactomycetes, Beta- and Gamma-proteobacteria, and Verrucomicrobia[10].

During atrophic gastritis, the stomach microbiota (Proteus mirabilis, Klebsiella pneumoniae, Staphylococcus aureus, Staphylococcus capitis subsp. urealyticus, and Micrococcus sp.) can reach densities at which its ureaseproducing members can cause a reaction in $13 \mathrm{C}$ urea breath tests, even in the absence of Helicobacter species. Similar effects have been reported during $\mathrm{H} 2$ receptor antagonist treatment, and in patients with compromised gastric mobility. The number of culturable bacteria increases with prolonged PPI (omeprazole) treatment duration. A 2-week treatment increased CFU counts of culturable bacteria in the stomach contents by a factor of about 200. PPI treatment over 12 months increases CFU counts by a factor of 10E6 [10].

Helicobacter pylori (discovered in 1983) is a carcinogenic pathogen, infecting the stomach of more than onehalf of all humans globally. H. pylori induces chronic inflammation of the stomach mucosa, without disease symptoms, that can complicate to gastric and duodenal ulcer disease, and malignant stomach tumours [9]. H. pylori infection results in decreased abundance of Lactobacillus spp., and increased bacterial diversity due to the presence of bacteria that are typically found in the lower intestinal tract.
Infection or colonisation of non-gastric parts of GIT affects the gastric microbiota and the immune reaction against microbiota components. Gastric microbial diversity, including multiple Lactobacillus spp. strains, are necessary to clear $\mathrm{H}$. felis infection. Co-infection with the nematode Heligmosomoidespolygyrus leads to reduced gastric pathology but fails to clear H. felis colonisation. The nematode infection induces a response dominated by anti-inflammatory TH2 T-cells, which reduces the pro-inflammatory TH1 T-cells response, induced by H. felis. Similarly, co-infection with enterohepatic Helicobacter species (H. bilis, H. hepaticus, or $\mathrm{H}$. muridarum) reduces the $\mathrm{TH} 1$ response and significantly reduces $\mathrm{H}$. pylori-induced gastric pathology (mediated by Treg T-cells). Conversely, co-infection with $\mathrm{H}$. hepaticus enhances the pro-inflammatory TH17 response, resulting in increased $\mathrm{H}$. pylori-induced gastritis [10].

H. pylori infection is usually acquired during early childhood. Adults rarely acquire $\mathrm{H}$. pylori. In old age, mucosal atrophy leads to a loss of H. pylori infection. H. pylori infection depends on chemotaxis (ChePep regulator), production of ammonia and increased $\mathrm{pH}$ (urease activity). While most infections result in asymptomatic chronic gastritis, some complicate to peptic ulcer, gastric adenocarcinoma, and mucosa-associated lymphoid tissue lymphoma. Rarely the human stomach can also be colonised by $H$. bizzozeronii, H. suis, H. felis, H. heilmanniisensu strict, and the enterohepatic $\mathrm{H}$. cinaedi. Bacterial numbers in the stomach have $24 \mathrm{hr}$ fluctuations (day-time mealtimes increases, and decreased at night). They increase with higher luminal pH (after treatment with PPI) [10]. The intestinal microbiota is composed of bacteria from different phyla, bacteriophages, and a single phylum of archaea, yeast, and filamentous fungi. Bifidobacterium, Bacteroidetes, Lactobacillus, Escherichia, Klebsiella, and Clostridium are the dominant bacteria. In children above 6 years and adults, the composition of intestinal microbiota variesconsiderably among individuals due to age, genetics, health status, geographical location, stress, antibiotics, and diet. Bacteroidetes and Firmicutes are most abundant as compared to Proteobacteria, Actinobacteria, Fusobacteria, Verrucomicrobia and Tenericutes. Actinobacteria and Bacteroidetes are abundant in children in rural Africa, whereas Firmicutes and Proteobacteria are abundant in European children. Prevotella, Xylanibacter, and Treponema are present only in African children. Enterobacteriaceae, abundant in European children, are not found in African children. The bacterial loads in different regions of the intestinal tract vary considerably. Bacterial load increases from the stomach, duodenum, jejunum, ileum, and the distal colon, where it is the highest. The human host benefits from the GIT microbiota by: fermentation (indigestible carbohydrates to absorbable SCFA), detoxification (bile acids and bilirubin), colonisation resistance (defence against pathogens), and a role in the development of the immune system. Colonisation resistance comprises the ability of GIT microbiota to deter pathogens from causing infections by blocking pathogens attachment, depleting nutrients, and preventing pathogenic bacteria from expressing virulence genes. Butyrate prevents $\mathrm{S}$. enterica from 
expressing T3SS, weakening epithelial invasion. Further, organic acids generated by intestinal microbiota alter luminal $\mathrm{pH}$ preventing growth and replication of pathogens, such as Salmonella spp. and Escherichia coli O157. Intestinal microbiota utilises most oxygen, creating anaerobic and capnophilic environment affecting gut colonisation [11]. Campylobacter jejuni is a commensal in a wide spectrum of hosts (birds and mammals), except for humans where it causes bacterial food-born gastroenteritis. Campylobacteriosis has a high prevalence worldwide and represent a significant socioeconomic burden. Campylobacteriosis develops after the ingestion of at least 500 viable bacteria. It is a self-limiting disease, that rarely leads to post-infectious complications, like Guillain-Barre syndrome (MillerFisher syndrome), reactive arthritis (Reiter's syndrome), post-infectious irritable bowel syndrome, and potentially immunoproliferative small intestinal disease. After ingestion, C. jejuni has to overcome the colonisation resistance that is maintained by the human intestinal microbiota. A fat-rich diet modifies the microbiota, increasing Firmicutes and Proteobacteria, while reducing Actinobacteria and Bacteroidetes, exposing the intestinal epithelial cells to C. jejuni adherence (TLR4 and NOD1/CARD4). In conditions of carbon excess, the major SCFA are acetate and formate, whereas in conditions of low carbon availability lactate and acetate. C. jejuni utilises acetate and lactate as a carbon sources. The major site of $\mathrm{C}$. jejuni inflammation is the colon, where it is transported across the epithelial barrier via M-cells. Acute mucosal inflammation, characterised by IFN-gamma, IL1-beta, IL6, IL8, IL12, and IL23 secretion, neutrophil infiltration, macrophage and DC activation, B- and T-cell proliferation. IgA and IgG contribute to the long-term protection, but they might be detrimental when cross-reacting with gangliosides in neurons (Guillain-Barre syndrome). TH1 and TH17 T-cells (IL17 and IL22) induce the innate responses and production of defensins in the epithelium, eradicating the pathogen [11]. Mice display colonisation resistance against this pathogen. Lactobacilli are predominantly found in the murine GIT, whereas HFA animals harbour higher loads of Enterobacteria (E. coli), Entrococci, Bacteroides / Prevotella spp. and Clostridia (Clostridium coccoides). Pro-inflammatory cytokines (TNF-alpha, IL6, and MCP1) are present in the colon of $\mathrm{C}$. jejuni-infected HFA mice. C. jejeunitranslocates to the MLN, but not other organs, or blood in gnotobiotic, or HFA mice. The commensal bacterial flora constitutes a barrier that effectively prevents colonisation and infection by pathogens [12]. Colonic microbiota is more complex, resulting in differences in concentrations of bacterial metabolites (short chain FA and ammonia). Probiotics
(Bifidobacteria and Lactobacilli) modulate intestinal commensals, maintaining the homeostasis, and conferring a health benefit on the host. Bifidobacterium breve, but not Lactobacillus casei, induces CD103+ DC-mediated development of IL10-producing TR1-cells. Oral B. breve administration ameliorates colitis in immunocompromised mice, inducing FOXP3+ Treg T-cells given naive CD4+ Tcells from WT mice, but not from IL10 KO mice [13]. L. casei LP1 strain, found in cheese, produces DHNA, 1,4dihydroxy-2-naphthoic acid that stimulates the growth of Bifidobacteria (human-originated B. longum subs. infantis KCTC3127, B. bifidum KCTC3202, and B. breve KCTC3220). DHNA produced by Propionibacteriumfreudenreichii ET3 strain can be commercially used as a prebiotic, a bifidogenic growth stimulator. DHNA is a precursor of menaquinone (vitamin K2), which functions as an electron transfer agent in the bacterial respiratory chain. DHNA improves DSS-induced colitis in mice, and reduces inflammation in IL10 KO mice [14]. Dietary arginine supplementation alters intestinal microbiota (a shift of Firmicutes to Bacteroidetes ratio to favorBacteroidetes in the jejunum and ileum), and activate innate immunity in mice. L-Arg is not only a building block for proteins, but also a precursor for the synthesis of many biologically active molecules, including NO, ornithine, polyamines (putrescine, spermine, and spermidine), creatine, and agmatine. Dietary supplementation with Arg improves endothelium-dependent relaxation, nutrient metabolism, immune responses, brown adipose tissue growth and development, and pregnancy outcomes in mammals. Arg supplementation reduces NF-kappa-B, ERK, and PI3K-AKT pathways, but activates p38 and JNK in jejunum and ileum, respectively. Argis a bsorbedin the proximal region of the small intestine. The small intestine of most mammals is the exclusive site for de novo synthesis of citrulline and Arg from glycine, glutamate and proline. Arg regulates the metabolism of intestinal bacteria (Lactobacillus in the jejunum, and Streptococcus in the ileum), and expression of TLR in enterocytes (TLR8 in the jejunum, and TLR4, TLR5, and TLR8 in the ileum). Arg supplementation enhances the expression of pro-inflammatory factors: increases the expression of IFN-gamma in the jejunum, and IL1-beta, IFN-gamma and TNF-alpha in the ileum. Arg supplementation promotes the expression of sIgA in jejunum and ileum; increases the expression of MUC2, MUC4, cryptidins 1,4 , and 5, cryptidin-related sequence 1c, and RNase agiogenin 4 in jejunum, and cryptidins 1, 4, and 5, CRS1C, REG3-gamma, LYZ2, and ANG4 in the ileum; and the expression of $\operatorname{sIgA}$ in the colon [15].

Fig. 1. Microbiota activates NF-kappa-B in GIT.Microbial colonisation of transgenic zebrafish (expressing GFP under the transcriptional control of NF-kappa-B) with commensal microbiota (Pseudomonas aeroginosa) activates NF-kappa-B and up-regulates its target genes in the intestinal and extra-intestinal tissues of the digestive tract. This activation requires the functional flagellar apparatus of P. aeroginosa. GIT is inherently more sensitive and a dominant site of host response to the microbiota, despite the exposure of all external surfaces to the surrounding microbial environment. Microbial community in the zebrafish intestinal lumen reaches higher densities (10E9-10 CFU/ml) than that of the surrounding water (10E4-5 CFU/ ml). TLR-initiated signals propagate through MYD88, p38 MAPK, JNK, IRF3, and NF-kappa-B. TLRs, or receptors for IL1 or 
IL18 regulate NF-kappa-B-dependent GIT innate immune response genes. 26 gene transcripts are differentially expressed in the GIT. A. Increased CFB expression in liver; B. Increased SAA expression in liver, swim bladder, and segment 3 of the intestine; C. Increased expression of MPO in neutrophils in the caudal hematopoietic tissue [16].
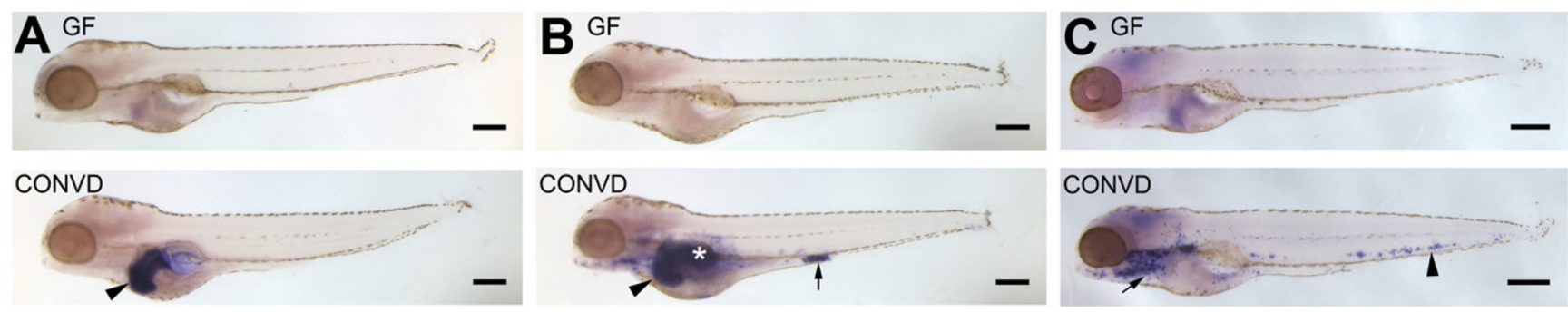

\section{CONCLUSION:}

Diet, diet supplementation, prebiotics, and probiotics along with genetic, epigenetic factors and environmental exposures influence in a forward way the development and function of the immune system. The individual's immune ecology, in a backward fashion, defined by the inflamatory (infection with EBV, CMV, HHV6, Chlamydia pneumoniae, Streptococcus pneumoniae, Saccharomyces cerevisiae), autoimmune (rheumatoid arthritis, multiple sclerosis, EAE, ulcerative colitis, asthma, diabetes), and neoplastic (lung, stomach, colon cancers) bodily status reflects in changes in the composition of the gastrointestinal microbiota.

Vaccine and vaccine adjuvants trigger the adaptive and innate immunity, sometimes leading to autoimmune and inflammatory syndromes that reflecs in changes in the microbiome. The development of mucosal vaccines, efficiently inducing immune exclusion via a non-inflammatory secretory IgA-mediated shielding of mucosal surfaces, easier to apply and better suited for mass administration depends on a better understanding of the mucosal (lung and gastrointestinal) immunity and the human microbiome.

\section{Abbreviations:}

Arg - arginine, BM - bone marrow, DC - dendritic cells, GC - germinal centre, GIT - gastrointestinal tract, HFA mice - human flora-associated mice, IBD - inflammatory bowel disease, IS - isotype switch, KO mice - knock-out mice, LPS - lipopolysaccharides, MALT - mesenteric autonomous lymphoid tissue, MLN - mesenteric lymph nodes, MR mice - microbial-reduced antibiotic-treated mice, NEC - necrotising enterocolitis, NO - nitric oxide, PP - Payer patches, ROS - reactive oxygen species, SCFA - short-chain fatty acids, WT mice - wild-type mice.

\section{Acknowledgements:}

The author would like to express gratitude to the staff of the Central Medical Library, Sofia, Bulgaria for the help during the preparation of this manuscript.

\section{REFERENCES:}

1. Dimmitt RA, Staley EM, Chuang G, Tanner SM, Soltau TD, Lorenz RG. Role of postnatal acquisition of the intestinal microbiome in the early development of immune function. J Pediatr Gastroenterol Nutr. 2010 Sep;51(3): 262-73. [PubMed] [CrossRef]

2. Lundell AC, Björnsson V, Ljung A, Ceder M, Johansen S, Lindhagen $\mathrm{G}$, et al. Infant B cell memory differentiation and early gut bacterial colonization. J Immunol. 2012 May 1; 188(9): 4315-22. [PubMed] [CrossRef]

3. Cahenzli J, Balmer ML, McCoy KD. Microbial-immune cross-talk and regulation of the immune system. Immunology. 2013 Jan;138(1):12-22. [PubMed] [CrossRef]

4. Herfel TM, Jacobi SK, Lin X, Fellner V, Walker DC, Jouni ZE, et al. Polydextrose enrichment of infant formula demonstrates prebiotic characteristics by altering intestinal microbiota, organic acid concentrations, and cytokine expression in suckling piglets. J Nutr. 2011 Dec;141(12): 2139-45. [PubMed] [CrossRef]

5. Rodríguez JM, Murphy K, Stanton C, Ross RP, Kober OI, Juge $\mathrm{N}$, et al. The composition of the gut microbiota throughout life, with an emphasis on early life. MicrobEcol Health Dis. 2015 Feb 2;26:26050. [PubMed] [CrossRef]

6. Tojo R, Suárez A, Clemente MG, de los Reyes-Gavilán CG, Margolles A, Gueimonde $\mathrm{M}$, et al. Intestinal microbiota in health and disease: role of bifidobacteria in gut homeostasis. World J Gastroenterol. 2014 Nov 7; 20(41): 15163-76.

[PubMed]

[CrossRef]

7. Jeffery IB, Lynch DB, O'Toole PW. Composition and temporal sta- bility of the gut microbiota in older persons. ISME J. 2016 Jan;10(1):17082. [PubMed] [CrossRef]

8. Jeong JJ, Kim KA, Jang SE, Woo JY, Han MJ, Kim DH. Orally administrated Lactobacillus pentosus var. plantarum C29 ameliorates agedependent colitis by inhibiting the nuclear factor-kappa B signaling pathway via the regulation of lipopolysaccharide production by gut microbiota. PLoS One. 2015 Feb 17; 10(2): e0116533. [PubMed] [CrossRef]

9. Heimesaat MM, Boelke S, Fischer A, Haag LM, Loddenkemper C, Kühl AA, et al. Comprehensive postmortem analyses of intestinal microbiota changes and bacterial translocation in human flora associated mice. PLoS One. 2012;7(7): e40758. [PubMed] [CrossRef]

10. Yang I, Nell S, Suerbaum S. 
Survival in hostile territory: the microbiota of the stomach. FEMS Microbiol Rev. 2013 Sep;37(5):73661. [PubMed] [CrossRef]

11. Masanta WO, Heimesaat MM, Bereswill S, Tareen AM, Lugert R, Groß U, et al. Modification of intestinal microbiota and its consequences for innate immune response in the pathogenesis of campylobacteriosis. Clin Dev Immunol. 2013;2013: 526860. [PubMed] [CrossRef]

12. Bereswill S, Fischer A, Plickert R, Haag LM, Otto B, Kühl AA, et al. Novel murine infection models provide deep insights into the "ménage à trois" of Campylobacter jejuni, microbiota and host innate immunity. PLoS One. 2011;6(6):e20953. [PubMed] [CrossRef]

13. Jeon SG, Kayama H, Ueda Y, Takahashi T, Asahara T, Tsuji H, et al. Probiotic Bifidobacterium breve induces IL-10-producing $\operatorname{Tr} 1$ cells in the colon. PLoSPathog. 2012;8(5): e1002714. [PubMed] [CrossRef]

14. Kang JE, Kim TJ, Moon GS. A Novel Lactobacillus casei LP1 Producing 1,4-Dihydroxy-2-Naphthoic Acid, a Bifidogenic Growth Stimulator. PrevNutr Food Sci. 2015 Mar;20(1): 78-81. [PubMed]

\section{[CrossRef]}

15. Ren W, Chen S, Yin J, Duan J, Li T, Liu G, et al. Dietary arginine supplementation of mice alters the microbial population and activates intestinal innate immunity. J Nutr. 2014 Jun; 144(6):988-95. [PubMed] [CrossRef]

16. Kanther M, Sun X, Mühlbauer M, Mackey LC, Flynn EJ 3rd, Bagnat $\mathrm{M}$, et al. Microbial colonization induces dynamic temporal and spatial patterns of NF- $\kappa \mathrm{B}$ activation in the zebrafish digestive tract. Gastroenterology. 2011 Jul;141(1):197-207. [PubMed] [CrossRef]

Please cite this article as: Hristov KK. Gastrointestinal Microbiota and Immunity. J of IMAB. 2019 Jul-Sep;25(3):26832688. DOI: https://doi.org/10.5272/jimab.2019253.2683

Received: 02/01/2019; Published online: 09/09/2019

\author{
Address for correspondence: \\ Kroum K. Hristov, \\ Department of Chemistry and Biochemistry, Medical University - Sofia, \\ Sofia - 1431, Bulgaria, \\ E-mail: 4747868@dir.bg,
}

